\title{
Algunas posibilidades de incorporación de la web 2.0 en la educación ${ }^{1}$
}

\author{
Gerzon Yair Calle-Álvarez² \\ gerzon.calle@udea.edu.co \\ Diego Alejandro Ocampo-Zapata ${ }^{3}$ \\ diego.ocampo@uniremington.edu.co
}

https://doi.org/10.22209/rhs.v7n1a05

Recibido: marzo 14 de 2019.

Aceptado: mayo 28 de 2019.

\section{Resumen}

El presente artículo de reflexión tiene como objetivo aportar a la discusión sobre la incorporación de las herramientas de la web 2.0 en la educación. Para ello, se comienza presentando las características de la web 2.0 de interactividad, sistema de gestión de contenidos, indexación. Se continúa planteando la relación existente entre la web 2.0 y el usuario, lo que permite desarrollar la idea de aprendizaje social. Para precisar el concepto en la perspectiva de una utilización didáctica, se hace una breve descripción de algunas herramientas y sus posibilidades de uso en la escuela: podcast, blog, wikis, webquest, redes sociales, conferencias electrónicas.

1 Las reflexiones que se presentan en esta ponencia hacen parte de los resultados de un trabajo de investigación sobre pensamiento crítico, escritura digital y web 2.0.

2 Integrante del grupo de investigación Didáctica y Nuevas Tecnologías de la Universidad de Antioquia.

3 Profesor de la Corporación Universitaria Remington.
Dentro de las conclusiones se encuentra que es oportuno que la escuela incorpore pedagógica y didácticamente, dentro de sus dinámicas institucionales, los recursos de la web 2.0 .

Palabras claves: Conferencia electrónica; Educación; Podcast; Red social; Webquest; Podcast; Web 2.0

\section{Abstract}

This reflective paper aims to contribute to the discussion on integrating web 2.0 tools into education. To this effect, it begins by presenting the characteristics of web 2.0, including interactivity, content management system and indexing. Then, the relationship between web 2.0 and the user is addressed, which allows the idea of social learning to be developed. To specify the concept of web 2.0 within the perspective of a didactic use, a brief description of some tools and their possibilities of use in the school is made: podcasts, blogs, wikis, web quests, social networks, electronic conferences. One of the conclusions reached by the research study is that it is appropriate for schools to pedagogically and didactically integrate web 2.0 resources into its institutional dynamics.

Keywords: Electronic conference; education; podcast; social network; webquest; Web 2.0. 


\section{Introducción}

L as tecnologías que se han ido ubicando a la vanguardia en cada época han repercutido fuertemente en las prácticas y reflexiones pedagógicas, y el modo de incorporarlas en la didáctica. Es por eso que, en algún momento, la imprenta se convirtió en el motor para la masificación de la lectura; la televisión se convirtió en un elemento transmisor de cultura; los componentes digitales en una posibilidad de trabajo colaborativo. Las Tecnologías de la Información y la Comunicación (TIC) se han transformado en un medio didáctico que puede propiciar la creación de entornos acordes con las necesidades vigentes de aprendizaje de los estudiantes; estas herramientas pueden estar orientadas a la construcción del conocimiento y aprendizajes significativos.

Los estudiantes actuales adquieren conceptos a través de las TIC y esos conceptos han sido tomados desde sus experiencias, ahora la tarea del profesor es potencializar esas ideas y fortalecerlas para que se conviertan en aprendizajes significativos. Como lo plantea Gallego-Badillo (2004), el aprendizaje significativo es una interrelación entre el nuevo conocimiento y los ya existentes, interacción en la cual ambos se modifican, la experiencia del joven se ve enriquecida gracias a las posibilidades que le brinda la escuela. El avance permanente de las TIC exige que la escuela no se estanque, de manera que las concepciones educativas y las prácticas pedagógicas se reconfiguren, sin embargo, existe la posibilidad de que las prácticas pedagógicas no evolucionen simultáneamente, creando un bache entre lo social y la escuela. Las perspectivas de las TIC, presentes para su uso educativo, exigen nuevos planteamientos que a su vez requerirán un proceso de reflexión sobre el papel de la educación en la sociedad, diferente al que nos tocó vivir como estudiantes, pero también provocarán un cuestionamiento de las instituciones educativas, para mirar si están preparadas para cumplir con los nuevos requisitos de formación, que está exigiendo la nueva sociedad.

En sus orígenes Internet fue pensado para asuntos de estrategias y trabajo. Con el tiempo esa mirada se ha ido ampliando y se han descubierto otras posibilidades y espacios de aplicación, y el usuario ha tomado mucha fuerza. En esos cambios la educación se ha visto inmersa, y parte de esas actualizaciones se debe a la web 2.0. De la Torre (2006) dice que la web 2.0 es una forma de entender Internet, donde la organización y el flujo de información dependen del comportamiento de las personas cuando acceden a ella.

Con Internet se abrieron otras posibilidades de ambientes de aprendizajes apoyados con TIC; sin embargo, en sus inicios, las posibilidades de incorporarlo en el aula fueron muy limitadas por falta de infraestructura tecnológica, recursos, falta de capacitación de los profesores o simplemente desconocimiento. El internet, en sus inicios, no fue pensado para la escuela, pero sus posibilidades fueron cautivando a la sociedad en general y la escuela comenzó a ver la necesidad de indagar y descubrir las posibilidades de este recurso. En la actualidad, se habla de escuela 2.0, atendiendo al concepto de las herramientas de la web 2.0. Poner este calificativo a la escuela no la hace 2.0, es necesario que se transformen y evolucionen otros aspectos de la escuela que están asociados a esos espacios de la web 2.0: la autonomía, la colaboración y la conectividad. 
La escuela está llamada a conectarse con el mundo. El aprendizaje, dejó de ser exclusivo de las paredes del salón de clases. Con las herramientas de la web 2.0, los profesores pueden participar en foros de cualquier parte del mundo, construir sus proyectos para trabajar en el aula; igualmente los estudiantes pueden explorar el planeta y descubrirlo por medio de videos o herramientas como Google Earth. La información ya no es exclusiva de unos pocos o restringida, es de todos y en esa medida se puede acceder a ella. En alusión a la autonomía, los ambientes de aprendizajes, apoyados por herramientas de la web 2.0, posibilitan fortalecer procesos de aprendizaje acorde con las realidades de los estudiantes. El estudiante traspasa los límites del tiempo y el espacio de clases para aprender, podrá construir sus conceptos, apoyarse en recursos visuales, auditivos y en algunos casos hasta táctiles para su aprendizaje, todas ellas asociadas a la toma constante de decisiones. En la colaboración, aunque existe una autonomía en el proceso de aprendizaje, los estudiantes no trabajarán solos, podrán apoyarse en las posibilidades de aprender con el otro, ya sea construyendo un texto en una wiki, mejorando sus argumentos por medio de un foro, preguntando a otros sobre su cultura, participando en procesos de etiquetado para identificar categorías.

Con la web 1.0 los procesos eran unidireccionales, los usuarios podían leer, escuchar, ver lo que las páginas les ofrecía. Con la web 2.0 ese rol de receptor se desvanece, así, los procesos de navegación y comunicación son bidireccionales, los usuarios pueden leer y escribir, escuchar y decir, ver y publicar sus propios videos $y_{\text {, }}$ además, poder modificar, comentar, participar, decidir frente a lo que está publicado (Calle-Álvarez, 2014). Este artículo de reflexión tiene como propósito aportar a la discusión de la incorporación de la web 2.0 en la educación, a la vez, permitirá a los profesores de los diferentes niveles educativos encontrar posibilidades de uso en las prácticas de aula.

\section{Uso didáctico de algunas}

\section{herramientas de la web 2.0}

Los CMS (Content Management System), o sistemas de gestión de contenidos, son una aplicación informática usada para crear, editar, gestionar y publicar contenido digital en diferentes formatos (Rodera y Barberà 2010), permite que las herramientas de la web 2.0 sean fáciles de manejar y asequibles, debido a que facilitan que un usuario sin necesidad de conocimientos en programación pueda publicar sus contenidos en la web; en algunos casos los CMS le dan al usuario plantillas de diseño, permitiendo a los usuarios concentrarse en la producción y evitando preocupaciones de bosquejos gráficos. Los estándares de sindicación de contenidos como los RSS (Really Simple Syndication), Atom, permiten el acceso a la información de manera rápida y sencilla, convirtiéndose en un aspecto clave al momento del flujo y distribución de la información. Los estándares de sindicación de contenidos se utilizan para la difusión de la información en donde se han suscrito los usuarios, pueden contener entradas, encabezados, artículos completos, resúmenes o enlaces a un sitio web.

La Folcsonomía, social bookmarking, indexación social o etiquetado social crea contenido de forma fácil, y permite desarrollar una clasificación colaborativa por medio de etiquetas simples o tag propuestas por los usuarios (Rodera y Barberà 2010). Así, los usuarios podrán clasificar los contenidos en categorías semánticas 
que posteriormente se convertirán en una herramienta para la recuperación y acceso a la información a través de una palabra clave. Las folcsonomías permiten que el usuario clasifique, almacene, ordene, comparta, busque y recupere los contenidos web de acuerdo con sus procesos de pensamiento (Pérez Sanchidrián, Campos Posada, y Campos Posada, 2015). Algunos sitios web que permiten el desarrollo de las folcsonomías son delicious, que permite etiquetar los enlaces favoritos; Flickr, indexación de fotos; Tagzania y Flof, etiqueta de lugares.

El usuario se convierte en el protagonista de la web 2.0, debido a ese componente social, donde el trabajo con el otro puede generar conocimiento y aprendizajes. La revista Time (2006), eligió como Personaje del año 2006, al usuario de internet, debido al impacto que ha tenido el usuario en la transformación de la web, donde se conjugan no solo lo tecnológico sino también lo social; las herramientas de la web proporcionan servicios, pero es el usuario a través de esos servicios que genera información, aprendizajes, conocimientos. Time (2006) destacó que los usuarios de internet adquirieron mayor poder para expresarse, son ciudadanos de la nueva democracia digital, son individuos que están cambiando la naturaleza de la era de la información, son los creadores y consumidores que están transformando el arte, la política y el comercio. El informe «Web 2.0. El Negocio de las Redes Sociales», publicado por la Fundación de la Innovación Bankinter, dice:

Gracias a las facilidades, evolución y gratuidad de tecnologías que antes eran inaccesibles para la mayoría de los usuarios, se están fomentando las redes sociales y la participación activa. El sitio web deja de ser un objetivo en sí mismo -el fin último de la interacción con el usuario-, para convertirse en una plataforma que posibilita la interrelación entre usuarios, miembros activos de una comunidad que comparte un interés o necesidad afín. Gracias a esto, el usuario tiene un papel activo: ya no sólo accede a la información, sino que además aporta contenidos y conocimiento (2007, p. 21).

Una de las posibilidades de la web 2.0 es la autonomía del estudiante. A través de los recursos el estudiante puede aprender, no necesariamente necesita una ruta dada por el profesor, él puede crear sus propias rutas de construcción del conocimiento, detenerse en alguna página para leer, comentar en otra, preguntar o responder en un foro, entre otras posibilidades. La autonomía se ve potencializada por otra posibilidad del uso de la web 2.0 y es el concepto de comunidad. Los estudiantes no se encuentran solos en los procesos de aprendizaje, pueden hacer parte de comunidades de aprendizajes o simplemente redes sociales (Martín-Moreno, 2004). En estos espacios, se aprende y se construye conocimiento con el otro, porque todos pueden aportar a la solución de un problema, la definición de un concepto, procesos de investigación, en estos espacios se socializan información, conocimientos, aprendizajes, recursos, preguntas que sus participantes van logrando.

El uso de las herramientas de la web 2.0 se convierte en una posibilidad para el aprendizaje social. Los estudiantes están inmersos en un mundo digital, donde elementos como los blogs, la mensajería instantánea, las wikis, se vienen convirtiendo en parte activa de sus procesos sociales y de aprendizaje. Sin embargo, no es únicamente el ser consumidor de información brindadas por páginas web, ahora los estudiantes han pasado a ser creadores de contenidos o parte de otros contenidos. Como afirman Tu, Blocher y Roberts (2008), el uso de la web 2.0 da la oportunidad a los estudiantes de actuar en un ambiente de aprendizaje que requiere 
que interactúen para lograr sus objetivos de aprendizaje. La web 2.0 ha permitido que los estudiantes presenten sus ideas a través de diversas herramientas. Inicialmente, los estudiantes consumían lo que unos pocos construían y publicaban en la red; en la actualidad, existen múltiples páginas libres, que ya tienen formatos preestructurados y la preocupación ya no está en procesos de programación sino en qué contenidos son los que se van a desarrollar, con qué características, para luego ser publicados.

La preocupación de publicar ya no es una responsabilidad en solitario. Las herramientas de la web 2.0 permiten un aprendizaje colaborativo, no se trata de sentarse a pensar sobre un asunto aislado del mundo, se trata de pensar un asunto conectado con el mundo (Rodera y Barberà 2010). Las redes sociales de aprendizaje son una forma de aprender con el otro y del otro. Cuando varias personas se unen con una finalidad de aprender el conocimiento generado, es un conocimiento social, donde los intereses que convocan al grupo se convierten en motivación para aprender y construir juntos. Vale anotar que la naturaleza del aprendizaje es social, y las herramientas de la web 2.0 permiten materializar esta idea, debido a la posibilidad de aprender en un entorno que se caracteriza por la autonomía, la interacción y la participación. Como dicen Tu et al. (2008). "Learners are engaged in contribution oriented pedagogy environments». La tecnología Web 2.0 tiene como objetivo mejorar la creación de conocimiento mediada por el intercambio de información, estructuras personalizadas, y, sobre todo, la colaboración entre los usuarios. «These concepts have led to the development and evolution of Web-based communities and hosted services, such as social networking sites, wikis, blogs, Second Life, and folksonomy».
La incorporación en el aula de las herramientas de la web 2.0, exige en los estudiantes diversos procesos de pensamiento, es decir, un estudiante al momento de enfrentarse al uso de estas herramientas debe pensar en lo que va a producir, en cómo lo va a producir y con quién o qué interacciones va a desarrollar para producir el contenido. Además, en la incorporación de estas herramientas en el aula, se hace necesario tener presente la dimensión metacognitiva del estudiante debido a las posibilidades de toma decisiones en la web 2.0. El estudiante permanentemente se verá enfrentado a modificar, copiar, pegar, cortar, agregar, conectar, quitar, relacionar, remezclar, dejar incompleto un contenido determinado, ya sea propio o de otro, con base en sus necesidades, intereses y capacidades. Tu et al. (2008) plantean que los entornos de la web 2.0 permiten a los estudiantes hacer visible su proceso de aprendizaje (modelos mentales) y ver otros procesos, además, faculta a los estudiantes de múltiples oportunidades para tomar el control de cómo van mejorado su forma de pensar y refinar sus estrategias metacognitivas. «It is the process of negotiating and managing how they would like to learn through self-reflection and assuming responsibilities».

La producción de textos en la web 2.0, van adquiriendo unas características propias. Los microcontenidos requieren de las capacidades de síntesis, claridad y precisión, con el propósito de dar la mayor información con pocas palabras, a la vez que se pueden convertir en una conexión a otros textos más amplios o en otros formatos. Esas conexiones, que se generan en los ambientes web 2.0, permiten en los estudiantes materializar sus procesos de pensamiento a través de rutas y decisiones que van tomando. Según Alexander (2008), escribir para una audiencia global es un poderoso estímulo para el cuestionamiento de la identidad personal, en 
representación de uno mismo a través de la escritura y la comprensión de un público; al reconocer que los procesos de escritura en la web 2.0 tienen como público una comunidad global, el estudiante trasciende los límites del profesor, ya no escribe para una nota o para ser leído por el profesor, ahora escribe para otros que le podrán aportar al texto, lo podrán modificar, enlazar o llegado el caso hasta eliminar.

Para precisar el concepto en la perspectiva de una utilización didáctica se hace una breve descripción de algunas herramientas que podrían apoyar el trabajo en el aula de clases.

Podcast. Es un archivo de audio distribuido mediante un archivo RSS, sin embargo, permite ser modificado o guardado en formato MP3, WAV, entre otros. Vale anotar, que con la evolución de la tecnología se pueden encontrar podcast de imágenes o videos. Los podcasts permiten que el estudiante puede escuchar sus descargas en cualquier momento, grabar conferencias y diálogos desde fuentes externas; es una herramienta útil para el trabajo de la expresión oral y la escucha en lenguas. Sin embargo, el podcast exige que el profesor invierta tiempo en colocar la información en la red, lo que implica profesores más comprometidos con el proceso formativo de los estudiantes y el propio, debido a que, si el profesor no tiene algunas nociones informáticas, la herramienta se le convertirá en un obstáculo más que en una posibilidad; lleva más tiempo elaborar un podcast, que publicar la información en un blog o en cualquier página web.

Hew (2009) presenta un estudio cuasi experimental, donde se utilizó el podcast como una herramienta de aprendizaje en un curso universitario y se comparaba con los estudiantes que no lo utilizaban. El autor le critica que no estaba bien definido el grupo control y que no hubo pre-test para definir el nivel inicial de los estudiantes. En la educación, el podcast, viene siendo subutilizado debido a que sus posibilidades se han limitado a guardar información oral, obviando las ventajas de la herramienta para fortalecer competencias comunicativas y cognitivas en las diferentes áreas del conocimiento.

Saborío Taylor, S. (2018) advierte sobre la necesidad de capacitar a los profesores en el uso de las TIC; en efecto, no se trata de implementarlas por un afán de innovación, ello lleva a una simple improvisación. Por ejemplo, el podcast no se aprovecha suficientemente debido a que, en la mayoría de los casos, el profesor no está capacitado para ello y, en consecuencia, no sabe cómo explotar todo el potencial que ofrece el podcasting. Igualmente, este autor afirma que las TIC deben estar integradas a la planeación metodológica y didáctica; para ello, el profesor debe asumir una responsabilidad en su formación continua en el uso e implementación de este tipo de tecnologías digitales.

Webquest. Es una herramienta para el trabajo de una unidad didáctica en cualquier área del conocimiento que consiste en un aprendizaje guiado con recursos principalmente procedentes de Internet, que promueve el trabajo colaborativo. Una webquest se construye alrededor de una tarea atractiva, se trata de hacer algo con la información de manera creativa o crítica, busca la solución de problemas, el análisis, la síntesis, el desarrollo de las habilidades mentales según la propuesta didáctica del profesor (Gallego Gil y Guerra Liaño, 2007). La tarea debe consistir en algo más que en contestar a simples preguntas o reproducir información. Según la propuesta didáctica del profesor deberá estar orientada a buscar la solución de problemas, el análisis, la síntesis, el desarrollo 
de las habilidades mentales, procesos de producción escritural. Sin embargo, lo que hay que tener presente es que la tarea debe consistir en algo más que en contestar a simples preguntas, reproducir información, copiar y pegar textos, se trata de hacer algo con la información: resumir, analizar, sintetizar, diagramar, transformar, crear, evaluar, buscando orientar el trabajo hacia la construcción del conocimiento y aprendizaje colaborativo.

Una webquest tiene la siguiente estructura: a) introducción, donde se hace una presentación general de la webquest; b) tarea, se presenta el resultado al finalizar la webquest; c) proceso, serie de actividades que se deben realizar para llegar o completar la tarea final; d) evaluación, criterios de valoración; e) conclusión, reflexiones u opiniones del tema; y orientaciones para el profesor, sugerencias para la aplicación y reproducción de la webquest en el aula. Vale anotar, que dentro del proceso están los recursos, orientados a las rutas para encontrar la información. Esta estrategia de aprendizaje dota a los profesores de las herramientas necesarias para usar las tecnologías de la información desde una perspectiva educativa, desarrollando sus propias ideas acordes con las temáticas que están enseñando. Supone una forma didáctica del uso del internet; permiten el trabajo interdisciplinar; fortalece el trabajo autónomo y colaborativo por parte de los estudiantes; Roig (2007) dice que las webquest son relativamente sencillas de planificar por el profesor, motivadoras para los estudiantes y fáciles de adaptar a las necesidades del aula. Dentro de las características de las webquest se encuentran: los estudiantes podrán hallar la mayor parte de la información en internet; su construcción permite que el proceso de aprendizaje sea guiado; orientada al desarrollo de competencias, habilidades o procesos de pensamiento; propone un trabajo motivador y factible para los estudiantes; fortalece el aprendizaje colaborativo.

Goig Martínez (2012) señala que el modelo constructivista implícito en la webquest «implica el fomento de interactividad», siempre y cuando se atienda a una variedad de recursos suficientemente diversos para acoger las numerosas experiencias y conocimientos de los sujetos implicados, es decir, variedad de recursos que permita un aprendizaje significativo por parte de los estudiantes, al tiempo que permita el aprovechamiento del saber específico del profesor y de sus estrategias metodológicas y didácticas. En palabras de la autora, de este modo la webquest constituye una poderosa herramienta que permite el incremento de la motivación, resolución de problemas y manejo de la información, el aprendizaje cooperativo, pensamiento crítico, lo que se verá reflejado en su desarrollo individual.

Wiki. Se les llama Wiki a las páginas Web con enlaces, imágenes y cualquier tipo de contenido que puede ser visitada y editada por diferentes usuarios desde cualquier lugar, se construyen a partir de un tema específico, de manera colectiva y permite a los usuarios crear, modificar o borrar el texto que comparten (Bernal Bravo y Trespaderne Arnaiz, 2015); de esta forma se convierte en una herramienta que permite crear textos colectivamente. El mayor uso de los wikis ha sido la creación de enciclopedias colectivas, por ejemplo, Wikipedia. Sin embargo, existen otros usos como creación de diccionarios, noticias, multimedias. Las wikis son fáciles de usar y aprender, no se necesitan de un editor o una persona que autorice la publicación de la información, democratizando lo que se publica y quiénes publican. La mayoría de los wikis guarda un historial de cambios lo que permite a los usuarios registrar todas las 
modificaciones que se vayan realizando; los estudiantes pueden trabajar acorde a su ritmo de aprendizaje. No obstante, debido a la naturaleza misma de los wikis cualquier persona puede editar, lo que puede ser demasiado abierto y generar vandalismo o información errónea, al mismo tiempo esta flexibilidad de la estructura de un wiki puede significar que la información se vuelve desorganizada.

En la educación, las wikis pueden ser utilizadas para desarrollar los conceptos de un área de conocimiento, elaboración de glosarios, construcción colectiva de contenidos, diseño de los propios textos, lo que implica que la responsabilidad de la wiki es de los estudiantes y el profesor. La aplicación de las wikis en la escuela puede fortalecer algunos procesos de aprendizaje colaborativo. Una investigación realizada por Wheeler, Yeomans y Wheeler (2008) con estudiantes universitarios de primeros semestres que utilizaron las wikis con regularidad durante las sesiones de aula como un espacio para almacenar y editar el trabajo de los ejercicios de su investigación, y como un foro de discusión, identificó algunas de las dificultades del trabajo en las actividades con las wikis, no lograron adaptarse a las preferencias de aprendizaje de todos los estudiantes; otra de las cuestiones que surgieron del uso de wikis en el aula fue el problema de la propiedad intelectual, debido al carácter colaborativo de la producción y donde no se evidencia los créditos personales. Si no existe una disciplina en el proceso de participación en la construcción de la wiki al final solamente unas pocas personas escriben y los demás solo leen.

Por otra parte, dentro de las bondades de las wikis que arroja el estudio se encuentra: una serie de habilidades de pensamiento de alto nivel podrían resultar del uso y gestión de wikis, las wikis ofrecen ambientes apropiados en la cual los estudiantes, que están separados geográficamente el uno del otro, pueden desarrollar los lazos sociales. Los estudiantes reportaron que fueron capaces de desarrollar sus habilidades de pensamiento crítico a través de la utilización de los espacios compartidos. Dentro de las conclusiones del estudio se encontró que la colaboración debe subrayarse como un objetivo clave de cualquier actividad wiki. Los estudiantes inicialmente pueden sentirse intimidados por la perspectiva de la escritura en la Web, y pueden experimentar ansiedad por recibir críticas de sus compañeros y de una audiencia web invisible, por ello, los profesores podrían mitigar estos temores mediante el uso de una simulación de la práctica que está abierta solo para el grupo de pares, antes de utilizar un espacio vivo wiki en línea. Los estudiantes deben entender que las publicaciones en las wikis no le pertenecen exclusivamente al autor porque es una propiedad colectiva.

Blogs. Es un sitio web que permite la publicación instantánea de entradas (post), recopilar textos, artículos, videos, enlaces, podcast y otros elementos que brinda la red de manera cronológica, apareciendo primero el más reciente, y donde el autor tiene la potestad de determinar qué se publica; además, permite a los lectores publicar comentarios sobre su contenido. Los blogs son actualmente uno de los fenómenos digitales con mayor fuerza en el mundo, tanto que se ha creado un campo semántico, con términos como: blogosfera, edublogs, blogroll, weblogger. Además, los «bloggers» han pasado de ser consumidores y observadores pasivos a ser personas activas en la creación de contenidos y culturas, permitiendo fortalecer una realidad que ha traspasado las barreras del lenguaje. 
Piscitelli (2005) afırma que el blog constituye un formato narrativo propio de la red. El diseño del blog permite las interacciones sociales y facilita la comunicación e interacción entre los autores y lectores del blog.

En algunas ocasiones las entradas se pueden etiquetar, permitiendo crear categorías para luego poder recuperar la información así no se encuentre en la página principal. Igualmente, se puede crear enlaces con otros blogs, páginas web, wikis u otros recursos para identificar el contenido sobre un tema y facilitar el acceso a los lectores que puedan estar interesados en el contenido referenciado. Para la creación de enlaces, existen dos posibilidades: permalink, es el enlace permanente que crea el autor del blog sobre otro blog para referenciarlo; el trackback o pingback que es un enlace inverso donde el autor de un blog se notifica en otro blog, que ha referenciado o comentado, de este modo avisa al otro blog que está siendo citado y automáticamente se le convierte a este en un permalink. El blog también permite crear un blogroll o lista de enlaces de otros blogs que tienen relación con el tema de blog, amplían el contenido del blog o simplemente son del agrado del autor del blog, funcionan como una lista de blog favoritos. A la vez, los programas de weblog permiten la sindicación, utilizando el título, encabezados y dirección permanente lo que hace que se puedan recuperar por medio de buscadores.

El blog ofrece un espacio personal, se pueden utilizar para fomentar en los estudiantes la escritura, para realizar autobiografías, discusiones sobre lecturas previas, pero, además, se puede solicitar opiniones o comentarios sobre lo publicado; puede servir para observar la evolución de los estudiantes en su proceso cognitivo, facilitando al profesor el seguimiento, debido a que la información se presenta cronológicamente. Por otra parte, debido a su naturaleza asincrónica no es necesario que los estudiantes realicen comentarios, respondan, argumenten de manera inmediata, tienen tiempo de reflexionar, buscar información para participar en el blog. Cuando se publica un blog el estudiante debe considerar que su audiencia no es solamente el profesor o sus compañeros, su público pasa a ser universal, ya que cualquier persona podrá leer o participar en el blog.

Churchill (2009) realizó una investigación sobre el uso de los blogs para la enseñanza y el aprendizaje en la educación superior, en una universidad de Hong Kong. Esta investigación hace parte de un proyecto que buscaba indagar sobre el uso de las herramientas de la web 2.0 en educación. La investigación se realizó con estudiantes de la Maestría en Tecnologías de la Información en la Educación, se diseñó un blog con el material de un curso y los estudiantes tenían acceso a él para consultar la información, generar ideas, resolver inquietudes. La pregunta de investigación fue ¿De qué manera un ambiente de blog complementa la enseñanza en el aula y da lugar a una experiencia mejor de aprendizaje?, dentro de los resultados se encuentran: la mayoría de los estudiantes manifestaron agrado por el apoyo del blog para el curso, la evaluación fue un motivador para la participación en los blog, los estudiantes manifestaron que fue agradable poder leer lo que sus compañeros escribían sobre las tareas asignadas, los blog permiten aprender de las observaciones y errores de los otros, sin embargo, los estudiantes expresaron que no había interés de continuar con el uso o diseño de blog por cuenta propia sin estar obligado a hacerlo. 
Investigaciones como las de Churchill (2009) concluyen que los blogs pueden ser útiles y eficaces en la educación debido a que permite leer los blogs de otros, recibir comentarios, vista previa y lectura de las tareas de los otros. Sin embargo, tener presente que al momento de trabajar con los blogs es importante regular las tareas de aprendizaje para presentar los resultados en él, enlazar la propuesta a la evaluación y que el profesor pueda orientar el trabajo desde su blog. Otros entornos de la web 2.0 como Youtube, podcast, pueden ser un recurso para ampliar las posibilidades del uso de los blogs en la escuela. Los desarrolladores de nuevas generaciones de sistemas de gestión de aprendizaje están comenzando a explorar las formas de integrar la web 2.0 con el fin de ofrecer sistemas que permitan mejorar el aprendizaje.

Wang y Woo (2010) realizaron un estudio exploratorio para investigar la promoción del pensamiento crítico en los estudiantes de la escuela secundaria mediante las reflexiones escritas en un blog. Para medir las habilidades del pensamiento crítico utilizaron el modelo de Newman. Las tres categorías del pensamiento crítico que se analizaron fueron: la experiencia, la relevancia y la justificación. El estudio encontró que los tipos de interacción con los compañeros y el profesor tuvo un efecto positivo mayor sobre el alcance del pensamiento crítico. Dentro de las conclusiones del estudio se encuentran: a) escribir reflexiones en línea utilizando el weblog tiene el potencial de promover el pensamiento crítico en los estudiantes; b) la naturaleza asíncrona de la bitácora permite más tiempo para que los estudiantes reflexionen y se refieran a otros recursos antes de publicar sus escritos.
Un estudio de Molina Alventosa, Valencia Peris y Suárez Guerrero (2016) muestra que los estudiantes perciben el blog como punto de interacción, es decir, como herramienta social que permite el aprendizaje. El mismo estudio advierte sobre la falta de interacción del profesorado en los comentarios; este punto reviste especial importancia, dado que lo que el estudiante percibe como ausencia de retroalimentación incide directamente en su proceso. Dentro de las conclusiones de la investigación se plantea que existe relación directa entre la percepción del estudiantado y los roles en la construcción del conocimiento arraigados en la universidad y opuesto, normalmente, en el uso de la web 2.0. Así, el uso del blog como herramienta didáctica influye no solo en la construcción de conocimiento, sino también en la relación pedagógica, afectando la percepción de la relación profesor-estudiante; esto último puede evitarse con un cambio de perspectiva respecto a la forma tradicional de concebir la relación pedagógica, en otras palabras, se trata de transformar la percepción jerárquica vertical de la relación profesor-alumno en un enfoque que permita una relación horizontal que permita la retroalimentación mutua.

Las redes sociales. No es una novedad que los asuntos donde se ven involucrados los intereses de los más jóvenes lleven a conclusiones ligeras por parte de los otros, en algún momento ese fue el caso del Rock and roll, la televisión y la moda. Los jóvenes suelen ser sometidos a críticas estrictas y si, además, viene relacionado con algo que no existía hace veinte años, las opiniones suelen resultar negativas, si a ello se le suma el fácil acceso a la tecnología esa situación resulta subvalorada, es el caso de las redes sociales, como Facebook. 
Las redes sociales vienen siendo subvaloradas no solamente por muchos adultos sino también por bastantes de sus usuarios. Es común escuchar algunos padres de familia decir: «Facebook es el culpable de que mi hijo no estudie», igualmente, es posible encontrar usuarios de las redes sociales que solamente las utilizan para jugar o enterarse de la vida de otros. Además, se puede descubrir profesores que prohíben el uso de Facebook o que todavía no lo han explorado. Huijser (2008) afirma que las tecnologías 2.0, y en particular los sitios de la red social ofrecen interesantes oportunidades para la escuela, pero que las aplicaciones educativas de estas tecnologías deben basarse en sólidos principios pedagógicos y conducidos por la investigación empírica y una cuidadosa evaluación, si se quiere lograr aprendizajes significativos en los estudiantes.

La historia ha demostrado como algunos elementos que han sido rechazados o excluidos de la escuela se han convertido en aliados. Un ejemplo del último siglo es la televisión, como un aparato que fue catalogado como promotor de la ignorancia en los niños. En la actualidad es utilizada en el aula de clases como un recurso para el apoyo de los procesos de aprendizaje, a través del uso de videos educativos, programas de televisión, películas que no fueron pensadas para la educación, pero que con la orientación adecuada se convierten en toda una estrategia didáctica para el fortalecimiento de la lectura, el pensamiento, la expresión, los valores, entre otras opciones.

Las redes sociales se pueden convertir en un aliado del aprendizaje (Colás-Bravo, González-Ramírez, y de Pablos-Pons, 2013), no se trata de crear perfiles y que los estudiantes se conecten entre sí, va mucho más allá. Es necesario diseñar ambientes de aprendizajes donde se evidencie un apoyo de las redes sociales. Esto obliga al profesor a asumir un rol orientador del proceso, reconocer que los estudiantes pueden descubrir y aportar otros asuntos que él no ha considerado, que el aprendizaje se da de manera colaborativa, que las estrategias de evaluación que venía desarrollando necesitan reconfigurarse. De otro lado, se hace necesario tener presente que las barreras del tiempo y el espacio no se limitan exclusivamente al aula de clases, un estudiante podrá desde otro espacio físico o virtual ampliar o proponer nuevas ideas, otros temas de discusión, terminar los argumentos que otros comenzaron.

En el ambiente de aprendizaje apoyado por redes sociales, la escritura digital, tiene un papel principal que se debe orientar. Allí confluyen elementos de alfabetización visual, pensamiento, metacognición. Los estudiantes deberán desarrollar habilidades cognitivas y metacognitivas en el proceso de construcción de sus textos, debido a que es un espacio donde se escribe para otros y con otros, y donde pueden confluir diversos modos para representar los pensamientos; fortalecer habilidades como la toma de decisiones, análisis de información, síntesis, entre otras, hacen parte de los retos. Valenzuela-Argüelles (2013) afırma que las redes sociales favorecen la publicación de contenidos, la autonomía, el trabajo en equipo, el acceso a diversas redes y la comunicación con expertos.

Como menciona Martín-Moreno (2004), las redes sociales como herramientas para el aprendizaje colaborativo incrementan la motivación de todos los estudiantes hacia los objetivos y contenidos del aprendizaje; favorece la retención de lo aprendido; promueve el pensamiento crítico al dar oportunidades a sus integrantes de debatir los contenidos; la diversidad de conocimientos y experiencias del grupo contribuye 
positivamente al proceso de aprendizaje, al tiempo que reduce la ansiedad que pueden provocar las situaciones individuales de resolución de problemas. Las redes sociales pueden ser unas aliadas en el proceso de aprendizaje o un distractor, ahí está el reto de la escuela para diseñar ambientes de aprendizaje apoyados por redes sociales donde se fortalezca el aprendizaje colaborativo y se pueda potencializar habilidades del pensamiento en los estudiantes.

Leal Ríos, García Leal, Estrada Bellman y Cabero Almenara (2018), en un estudio sobre los hábitos de comunicación en las redes sociales virtuales por estudiantes de la Universidad Autónoma de Tamaulipas (México), identificaron la existencia de grupos de estudiantes sin poder adquisitivo para contratar un servicio de internet domiciliario. Los datos varían de un país a otro, y aún de una institución a otra, por ello es necesario tener en cuenta si los estudiantes pueden acceder, o no, a internet desde su domicilio o solo desde la institución. El saber cómo acceden los estudiantes, y desde qué dispositivo, es crucial para planear estrategias $\mathrm{O}$ actividades relacionadas con las TIC. Por otra parte, el estudio plantea que Facebook y WhatsApp son muy utilizados por los estudiantes, y lo hacen por el celular, datos que deben ser considerados a la hora de diseñar, o planear, estrategias educativas que impliquen las redes sociales. Por último, debe considerarse si el uso de estas redes obedece a un proyecto institucional, iniciativas individuales de profesores o alumnos.

Conferencias electrónicas. En la era de las TIC muchas de las discusiones se dan en espacios virtuales. Un periodista podrá publicar desde una perspectiva cierto acontecimiento y lo más seguro es que reciba un contraargumento sobre esa posición de un lector, u otro periodista que tiene la otra parte de la historia. Esto también puede ocurrir en un chat, un foro, un blog, una conferencia electrónica, entre otras; espacios que no solamente generan información, sino que se pueden convertir en posibilidades de crear discusiones en torno a puntos de vistas y opiniones. La conferencia electrónica, también llamada videoconferencia, e-conferencia o conferencia web, es una herramienta que posibilita que varias personas puedan ver y escuchar sobre un tema, a la vez que participar sobre el mismo. El soporte de la conferencia puede ser el correo electrónico, el chat, los foros, ya que a través de estos medios se podrá enviar y recibir información sobre la conferencia, sin embargo, todos los mensajes con destino a la conferencia quedarán registrados en el sitio web de la conferencia correspondiente. Por lo tanto, la conferencia electrónica podrá desarrollarse de manera sincrónica o asincrónica.

Entre las características de la conferencia sincrónica se encuentran: a) no es necesario que los participantes estén presentes en el mismo espacio geográfico, debido a que el espacio virtual los agrupa, sin embargo, sí es necesario que los participantes se encuentren presente a la misma hora geográfica desde donde se está generando la conferencia, eso implica que las diferencias horarias pueden ser un obstáculo para que la conferencia llegue a más público; b) se puede utilizar el chat como herramienta para participar en la conferencia de manera inmediata, por lo tanto, se podrá obtener respuesta de manera instantánea sobre las inquietudes que se van generando por parte de los expertos o por parte de otro participante.

En cuanto a las características de la conferencia electrónica asincrónica, se pueden nombrar: 
a) las personas pueden interactuar con otras personas sin importar el espacio geográfico o el tiempo, así, un europeo podrá participar en la misma conferencia que un americano, a pesar de las distancias geográficas y diferencia horaria, porque al ser una comunicación asincrónica no será necesario que los participantes estén al mismo tiempo para participar, se podrá hacer en el momento que la persona pueda ingresar a la conferencia y responder a las preguntas que le plantearon, generar nuevas inquietudes, cerrar ideas, etc. b) Los participantes pueden descargar los textos del debate, los videos, el audio, en general el material virtual disponible de la conferencia durante y después de la conferencia, lo que permitirá que los participantes pueden revisar la información las veces que sea necesario para mejorar su comprensión, incluso, descargarla a dispositivos móviles.

En una investigación realizada por Coffin, North, y Martin (2008), se presentan algunas herramientas para trabajar en la básica secundaria la argumentación apoyados en la conferencia electrónica asincrónica, además, en el estudio se realizó un análisis desde la Lingüística Sistémico Funcional. Los autores plantean en el estudio que: a) al no estar limitada las relaciones espacio tiempo, la conferencia electrónica asincrónica permite que se puedan identificar diversos tipos de argumentos y contraargumentos debido a las diversas miradas de los participantes, así los argumentos podrán ser pensados y analizados antes de ser planteados a los grupos; b) la comprensión de las conferencias podrá ser mayor debido a que los participantes podrán revisar los contenidos las veces que sean necesarias esto beneficiará el proceso de creación de puntos de vistas.

Dentro de las conclusiones planteadas en la investigación se cita el potencial de la herramienta para el desarrollo de la argumentación en la escuela, sin embargo, es importante tener presente que durante la conferencia electrónica el rol del profesor es fundamental para crear «conflictos socio cognitivos» (Piaget, 1980) y así generar las posibilidades de procesos de construcción de argumentos válidos alrededor de un tema. Igualmente, el profesor podrá ser un guía o un obstáculo dentro del proceso de la conferencia, si el profesor interviene para motivar las discusiones u orientarlas, el proceso puede dar resultados, por el contrario, si el profesor desde un comienzo limita a los estudiantes, marca o reprocha a los estudiantes sobre sus intervenciones, lo más seguro es que la participación sea limitada por los temores de ser sancionado. Los beneficios de la conferencia electrónica asincrónica para desarrollar la argumentación están enmarcados en los procesos de reflexión, negociación, discusión, aprendizaje colaborativo, autonomía, autocorrección que se pueden dar durante los procesos de construcción de los argumentos.

\section{Conclusiones}

Las herramientas podcast, webquest, wiki, blog son una posibilidad para fortalecer los procesos de enseñanza y aprendizaje en la escuela, sin embargo, no hay que ponerlos como la solución a los problemas formativos, sino como un camino para incorporar herramientas de la web 2.0 al aula y permitir el trabajo colaborativo, autónomo y democratizado por parte de los profesores y los estudiantes. Al reconocer las herramientas de la web 2.0 como espacios auténticos para la producción de textos existe una posibilidad para que los estudiantes puedan fortalecer sus procesos de escritura digital, además, en estos entornos se hace necesaria la aplicación de habilidades de pensamiento crítico como la toma de decisiones, la solución 
de problemas, la reflexión para poder generar productos que sean leídos, comprendidos, contraargumentados por los usuarios de la red.

Las herramientas podcast, webquest, wiki, blog, conferencia electrónica y cualquier otra de la web 2.0 que se decida considerar o implementar en la escuela requiere que sea analizada como un recurso, es decir, no basta solo con incorporar la tecnología, es necesario que se revisen las condiciones contextuales y didácticas para que su uso tenga repercusiones en los aprendizajes de los estudiantes. Por ejemplo, el podcast es un recurso adecuado para fortalecer la oralidad, sin embargo, requiere de toda la estructura didáctica para que sea adecuado para lograr este objetivo.

No será suficiente con poner el calificativo de escuela 2.0, se hace necesario pensar desde el contexto cada uno de los componentes del ambiente de aprendizaje para poder construir propuestas innovadoras apoyadas por TIC en la escuela. Como dice Bryant (2007), la adopción de herramientas, técnicas e ideas de software social será el ejemplo más importante y visible del empleo de la tecnología emergente en educación en los próximos años, pero son los logros sociales, no la tecnología en sí, lo que resulta novedoso y excitante.

En la web 2.0, la imagen y los enlaces aportan a la construcción de significados de los textos digitales. La imagen tiene la posibilidad de actuar para ejemplificar, ampliar, precisar, comparar las ideas del autor dentro de la construcción de su posición, argumento o conclusión. Los enlaces, permiten conectar el texto con otros textos que guardan cierta relación entre ellos y que actúa como una ruta para precisar las ideas, contrastar opiniones, ampliar argumentos, definir palabras, ampliar el campo de acción, cerrar discusiones.
La implementación de un ambiente de aprendizaje apoyado con herramientas de la web 2.0, permite que los estudiantes compartan opiniones con los compañeros de grupo, conozcan las ideas de otras personas sobre el tema, compartan y despiertan el interés sobre discusiones de temas sociales. Igualmente, las sesiones de trabajo estimulan los procesos de escritura y creatividad en los estudiantes, contribuyen a comprender los textos consultados y crea consciencia en los estudiantes de cada uno de los temas en discusión.

Cuando se determina incorporar alguna herramienta de la web 2.0 a los procesos de enseñanza y aprendizaje, el profesor deberá tener un objetivo didáctico asociado a los saberes disciplinares, además, identificar cuáles son los aportes diferenciadores de la herramienta, en la construcción de conocimiento. El uso de la tecnología en la educación por sí sola no la convierte en una práctica innovadora ni asegura el aprendizaje significativo, se debe partir de la realidad institucional y los saberes previos de los estudiantes. A partir de ahí, se debe determinar la ruta de trabajo y la forma de incorporación y desarrollar el valor agregado de la tecnología.

\section{Referencias}

- Alexander, B. (2008) Web 2.0 and emergent multiliteracies. Theory Into Practice 47,150-160. doi: 10.1080/00405840801992371

- Bernal Bravo, C. y Trespaderne Arnaiz, G. (2015). Wikis en la Enseñanza Secundaria. IJERI: International Journal of Educational Research and Innovation, (3), 52-63. Recuperado de https:// www.upo.es/revistas/index.php/IJERI/article/ view/1443 
- Bryant, L. (2007). Emerging trends in social software for education. En: British Educational Communications and Technology Agency Emerging Technologies for Learning: Volume 2. Coventry: Becta.

- Calle-Álvarez, G. (2014). Las habilidades del pensamiento crítico durante la escritura digital en un ambiente de aprendizaje apoyado por herramientas de la web 2.0. Revista Encuentros, Universidad Autónoma del Caribe, 12(1), 27-45. doi: http://dx. doi.org/10.15665/re.v12i1.200

- Colás-Bravo, P., González-Ramírez, T., y de Pablos-Pons, J. (2013). Juventud y redes sociales: Motivaciones y usos preferentes. Comunicar: Revista Científica de Comunicación y Educación, 20(40), 15-23. doi https://doi.org/10.3916/ C40-2013-02-01

- Coffin, C., North, S y Martin, M. (abril 2008) Exchanging and countering points of view: a linguistic perspective on school students' use of electronic conferencing. Journal of Computer Assisted Learning 25, 85-98.

- Churchill,D. (2009). Educational applications ofWeb 2.0: Using blogs to support teaching and learning. British Journal of Educational Technology, 40(1), 179-183. doi: 10.1111/j.1467-8535.2008.00865.x

- De la Torre, A. (enero 2006). Web Educativa 2.0. Revista Electrónica de Tecnología Educativa (20). Recuperado de: http://www.uib.es/depart/gte/gte/ edutec-e/revelec20/anibal20.htm

- Fundación de la Innovación Bankinter (2007) Web 2.0. El Negocio de las Redes Sociales. Recuperado de: http://www.fundacionbankinter.org/es/ publications

- Gallego-Badillo, R. (2004). Discursos constructivistas sobre las tecnologías. Una mirada epistemológica. Bogotá: Magisterio.
- Gallego Gil, D. y Guerra Liaño, S. (junio 2007). Las WebQuest y el aprendizaje cooperativo. Utilización en la docencia universitaria. Revista Complutense de Educación, 18(1), 79. Recuperado de http:// search.proquest.com/openview/b1fb0b46402b2ff3608102fd1c7ed230/1?pq-origsite=gscholar

- Goig Martínez, R. A. (2012) El Uso de la Webquest como Recurso Didáctico Innovador en el $2^{\circ}$ Ciclo de Educación Infantil. Revista Electrónica de Investigación y Docencia (REID), (7), 73-89. Recuperado de https://revistaselectronicas.ujaen.es/ index.php/reid/article/viewFile/1089/920

- Hew, K.F. (2009) Use of audio podcast in K12 and higher education: a review of research topics and methodologies. Educational Tech research Development 57(3), 333-357.

- Huijser, H. (2008) Exploring the Educational Potential of Social Networking Sites: The Fine Line between Exploiting Opportunities and Unwelcome Imposition. Studies in Learning, Evaluation, Innovation and Development, 5 (3). Recuperado de: http://eprints.usq.edu.au/4426/2/Huijser_2008_ Sleid.pdf

- Leal Ríos, F., García Leal, M., Estrada Bellman, P. C., y Cabero Almenara, J. (2018). Uso de las redes sociales virtuales por los estudiantes de la UAT. Revista de Ciències de l'Educació, 1, 32-42. Recuperado de https://idus.us.es/xmlui/handle/11441/78279

- Martín-Moreno C. Q. (2004) Aprendizaje colaborativo y redes de conocimiento. Libro de actas de las IX Jornadas Andaluzas de Organización y Dirección de Instituciones Educativas. Granada. Grupo Editorial Universitario, 55-70. http://www. ugr.es/ sevimeco/UGR/biblioteca/orgeduc/redes/Quintina\%20Martin\%20Moreno.pdf 
- Molina Alventosa, J. P., Valencia Peris, A., y Suárez Guerrero, C. (2016). Percepción de los estudiantes de una experiencia de uso didáctico de blog docente en Educación Superior. Educación XX1, 19(1). Recuperado de http://revistas.uned.es/index.php/educacionXX1/article/view/15579

- Pérez Sanchidrián, E., Campos Posada, R. y Campos Posada, G. E. (2015). Etiquetado social: un modelo de representación de la información en la blogosfera. Biblios: Revista de Bibliotecología y Ciencias de la Información, (56), 19-27. Recuperado de http://biblios.pitt.edu/ojs/index.php/biblios/ article/view/187

- Piaget, J. (1980) Psicología y pedagogía. Barcelona: Ariel.

- Piscitelli, A. (2005) Internet. La imprenta del siglo XXI. Barcelona. Gedisa.

- Rodera, A., y Barberà, E. (2010). LMS y web 2.0 una relación simbiótica en las aulas universitarias Diseño e integración de actividades pedagógicas 2.0 en una plataforma Blackboard. Revista de Educación a Distancia, 2, 1-27 Recuperado de http://revistas.um.es/red/article/view/244341/0
- Saborío Taylor, S. (2018). Podcasting: Una herramienta de comunicación en el entorno virtual. Innovaciones educativas, 20(29), 95-103. Recuperado de: https://dialnet.unirioja.es/servlet/ articulo?codigo $=6719663$

- Time (2006) Personaje del año 2006. Recuperado de: http://www.time.com/time/magazine/article/0,9171,1570810,00.html

- Tu, C., Blocher, M., y Roberts, G. (2008). Constructs for Web 2.0 learning environments: A theatrical metaphor. Educational Media International, 45(3), 253-268. doi: 10.1080/09523980802588576

- Valenzuela-Argüelles, R. (2013). Las redes sociales y su aplicación en la educación. Revista Digital Universitaria UNAM, 14(4). Recuperado de: http://www. revista.unam.mx/vol.14/num4/art36/art36.pdf

- Wang, Q y Woo, H. (2010) Investigating students' critical thinking in weblogs: an exploratory study in a Singapore secondary school. Asian Pacific education review 17(4), 541-551.

- Wheeler, S., Yeomans P., Wheeler, D. (2008) The good, the bad and the wiki: Evaluating student-generated content for collaborative learning. British Journal of Educational Technology, 39(6), 987-995. 